\title{
Modeling and optimizing an agro-supply chain considering different quality grades and storage systems for fresh products: a Benders decomposition solution approach
}

\author{
Fatemeh Keshavarz-Ghorbani ${ }^{1}$. Seyed Hamid Reza Pasandideh ${ }^{1}$
}

Accepted: 17 August 2021 / Published online: 4 October 2021

(c) The Author(s), under exclusive licence to Springer Science+Business Media, LLC, part of Springer Nature 2021

\begin{abstract}
This paper proposes a mathematical model in the context of agro-supply chain management, considering specific characteristics of agro-products to assist purchase, storage, and transportation decisions. In addition, a new method for determining the required quality score of different types of products is proposed based on their loss factors and purchasing costs. The model aims to minimize total cost imposed by purchasing fresh products, opening warehouses, holding inventories, operational activities, and transportation. Two sets of examples, including small and medium-sized problems, are implemented by general algebraic modeling language (GAMS) software to evaluate the model. Then, Benders decomposition (BD) algorithm is applied to tackle the complexity of solving large-sized instances. The results of both GAMS and BD are compared in terms of objective function values and computational time to demonstrate the efficiency of the BD algorithm. Finally, the model is applied in a real case study involving an apple supply chain to obtain managerial insights.
\end{abstract}

Keywords Agro-supply chain · Quality score · Benders decomposition algorithm • Inventory management

\section{Introduction}

Over recent decades, agricultural logistics has drawn the attention of researchers and practitioners due to increasing concerns in economic, healthy feeding, environmental, and political issues. Hence, good management in post-harvest operations, such as

Seyed Hamid Reza Pasandideh

shr_pasandideh@khu.ac.ir

Fatemeh Keshavarz-Ghorbani

std_fatemeh_kgh@khu.ac.ir

1 Department of Industrial Engineering, Faculty of Engineering, Kharazmi University, Tehran, Iran 
packaging, storing, and distributing agro-products, can add significant value to logistics while satisfying customers' demands and maintaining food security. Therefore, research on agricultural logistics can speed up modernization and competition abilities in agricultural processes (Otchia 2014).

The specific characteristic of agro-products, such as seasonality, perishability, and long procurement time, make it different from other supply chains, therefore, it is necessary to have accurate information about their effects on supply chain management. These characteristics put restrictions on logistic decisions and make deep concerns over resource depletion and the occurrence of agricultural wastes and losses. According to Widodo et al. (2006), 20-60\% of agro-products are wasted along supply chains in any country. Therefore, designing efficient inventory and transportation systems for perishable products plays a vital role in cost-saving and freshness-keeping (Coelho and Laporte 2014). From this perspective, cold chains with proper temperature adjustment can significantly reduce losses (Yu and Xiao 2017).

Although efficient agro-supply chain management prevents storage and transportation losses, facility location problem is another issue strongly affecting the security and safety of foods, especially in developing countries (Morton et al. 2008). Given capacity constraints in warehouses, determining warehouse locations and assigning cold storage rooms under controllable temperature conditions play a pivotal role in loss and waste reduction over a supply chain that may yield a complicated problem (Aung and Chang 2014). Forasmuch as the current study deals with agricultural products with a short shelf life, optimal facility location and storage condition decisions can reduce losses due to the dispersed distribution centers, long distances transportation, etc.

This study presents an agro-supply chain based on a case study involving a wide range of decisions such as supplier selection, facility location, inventory control, and transportation planning. This research aims to optimize the shelf life of products via assigning proper cold storage systems that can prevent postharvest losses. In addition, different quality grades are considered for each type of product, whereby those farmers who have gotten the average quality score are selected to satisfy warehouses' demands. For these purposes, a mixed-integer nonlinear programming (MINLP) problem is formulated considering several operational constraints. Then, the model is linearized and transformed to mixed-integer linear programming (MILP) problem and solved by GAMS/CPLEX solver in small and medium sizes. Moreover, Benders decomposition (BD) algorithm is proposed to tackle the complexity of the problem caused by increasing the number of binary and integer variables in large sized-instances. Then, the proposed model is implemented for a real case study in Iran covering eight months of a year.

The rest of the paper is organized as follows: Sect. 2 gives an overview in the context of agro-supply chains. In Sect. 3, problem definition, notations, assumptions, and the mathematical model are presented. In Sect. 4, BD algorithm is proposed to tackle the complexity of large-sized instances. In Sect. 5, numerical experiments and a case study are performed to demonstrate the practicality of the proposed model. Section 5 draws conclusions and remarks on some future research directions. 


\section{Literature review}

Economic costs in agro-supply chains have been implied by inefficient management in purchasing, storage, and distribution of agro-products (Ahumada and Villalobos 2009). Although there are copious studies dealing with facility location and transportation problems, they have not been widely discussed for agricultural products. The facility location problem is a critical element of strategic decisions in agro-supply chains, especially in developing countries (Morton et al. 2008). This is because transportation and storage time will become shorter by properly determining facility locations (Morganti and Gonzalez-Feliu 2015). Therefore, dealing with the capacitated facility location problems with applications in storing and distributing agro-products is an essential component to adapt to environmental and competitive aspects (Validi et al. 2014).

Here, related literature with emphasis on three core parts of agro-supply chains, purchasing, facility location, and transportation decisions, has been reviewed to pinpoint the research gap. In this context, Paam et al. (2016) and Ganesh Kumar et al. (2017) have comprehensively reviewed agro-supply chain models. Then, Banasik et al. (2017) analyzed tradeoffs between economic and environmental benefits of an agro-food closed-loop supply chain with location-allocation decisions and production planning. They attempted to valorize agro-wastes by applying recycling concepts. Ghezavati et al. (2017) proposed a mathematical model for a distribution planning system considering the freshness and ripeness of products. They proposed BD algorithm to solve their problem in a reasonable time. Orjuela-Castro et al. (2017) presented a MILP model for a multi-product facility location problem. They aim to minimize the costs of transportation, installation, and fruit losses and show how intermediate logistics platforms affect fruit losses. Soto-Silva et al. (2017) formulated a two-stage agro-supply chain model for a case study in Chile. The first stage contains particular decisions on supplier selection and transportation planning and the second stage associates with establishing different types of cold chambers to store products and transportation planning. Allaoui et al. (2018) proposed a two-stage programming model for a sustainable agro-food supply chain. In the first stage, a hybrid analytic hierarchy process with an ordered weighted averaging method was used for assigning weights to sustainable indicators. In the second stage, a multi-objective mathematical model was proposed with respect to the results in the first stage. Paam et al. (2018) proposed an agro-supply chain model to optimize the shelf lives of apples with different quality grades in cold storage rooms. Liu et al. (2018) studied optimal purchase decisions and inventory retrieval policies for a single-product finite period agro-supply chain to maximize total profit under fluctuating prices. Jonkman et al. (2019) investigated the impacts of location-allocation, inventory, and transportation decisions on an agro-food supply chain under uncertain conditions. Liu et al. (2019) developed a transportation model for fresh agricultural products considering the impacts of ethephon concentration, time, and temperature on the maturity of soluble solids. They used ant colony algorithm to solve their model. Onggo et al. (2019) assessed stochastic demand in a multi-period inventory routing problem and attempted to minimize inventory, transportation, and shortage costs. They used a local metaheuristic algorithm to tackle the complexity of their model. Paam et al. (2019) proposed a new model for inventory management 
optimization in an apple supply chain. They analyzed the effects of two scenarios in storage rooms on fruit losses. Yu et al. (2019) investigated the impacts of horizontal and vertical integration on the quantity/quality losses and retailer profits.

The prior work on agro-supply chains is summarized in Table 1 to reveal existing gaps in the literature. The majority of the research has investigated multi-level supply chains under several constraints, without sufficient consideration on specific characteristics of agro-products, farmers, and distribution processes. However, a survey of practical experiences illustrates significant impacts of these properties on spoilage rate, in turn, on the amount of loss and system cost. Therefore, this paper takes several actions to fill the gaps, which are vividly explained as follows:

This study is an extension of the work done by Soto-Silva et al. (2017) in a way to be applicable for an apple supply chain in Iran. In their study, unlike many agro-supply chain models, three types of refrigeration technologies for maintaining different types of agro-products have been proposed that can make a privileged contribution in this context. In spite of their primary step towards sustainability of agro-products, it lacks an in-depth discussion on adjustment of storage conditions and its effects on inventory decisions. However, practical accessibility gave us valuable insights to design a mathematical model for adjusting storage conditions according to costs imposed on the system and its effects on the durability of agro-products. In addition, the storage of agro-products for a long-time mainly depends on their initial quality. Therefore, this study aims to manage the quality of agro-products by giving scores to producers and accordingly selecting them. To the best of the authors' knowledge, there is no previous effort on quality management despite its importance in handling the shelf life of agro-products. Overall, the main contributions of the paper are as follows:

- A MINLP model has been presented to jointly optimize facility location, transportation, inventory decisions in an agro-supply chain.

- A new method is proposed to assign weights to suppliers based on their loss factor. Accordingly, decisions on supplier selection are made.

- The current research seeks to optimize the shelf life of products through adjusting appropriate storage conditions.

- The proposed MINLP method is linearized and transformed to a MILP model.

- BD algorithm has been utilized to tackle the complexity of the proposed model and solve it in a reasonable time.

- The proposed model is implemented in a real case study in Iran to demonstrate its applicability and efficiency.

\section{Problem definition}

This study is an extension of the proposed model by Soto-Silva et al. (2017), who addressed supplier selection, transportation, and facility location decisions in an agro supply chain. However, their study lacks in-depth discussions on quality management and storage and inventory planning. Therefore, we formulate a multi-period MINLP model under some operational constraints to intertwine supplier selection, transportation, storage operation, inventory planning, and facility location decisions 


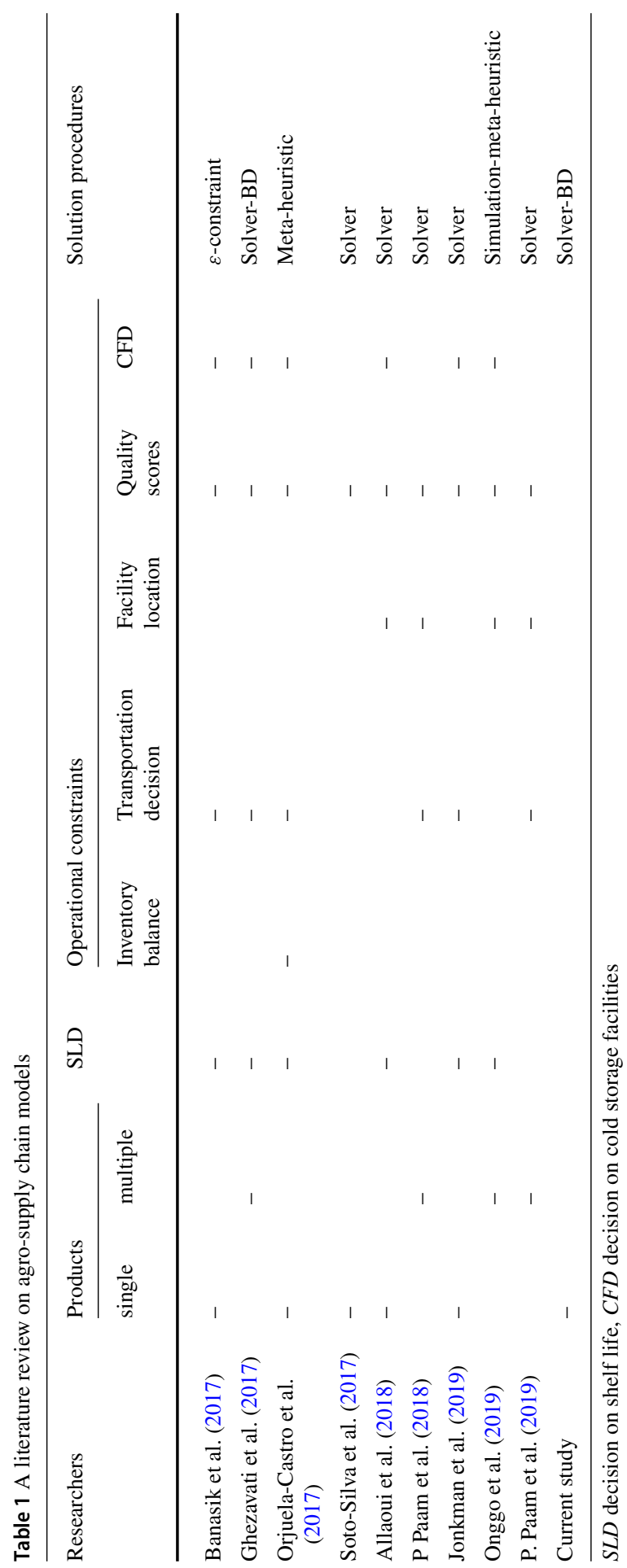


in an agro-supply chain. Afterwards, we reformulate it to a MILP model and propose BD algorithm to solve it. Figure 1 is meant to show the process of this paper.

Figure 2 is a perspective of the proposed agro-supply chain, including producers, warehouses, and a wholesaler. Fresh products should be purchased from the producers who gain the required score based on their quality grades. This approach satisfies the required quality of products to be kept for a long time in cold chambers, which is more profitable for the system. The products are shipped to the warehouses by the private fleet. The reason is that producers, either small or medium sizes, are often located in rural and sparsely populated areas. The long distances between orchards and facilities

Fig. 1 Overview of the paper
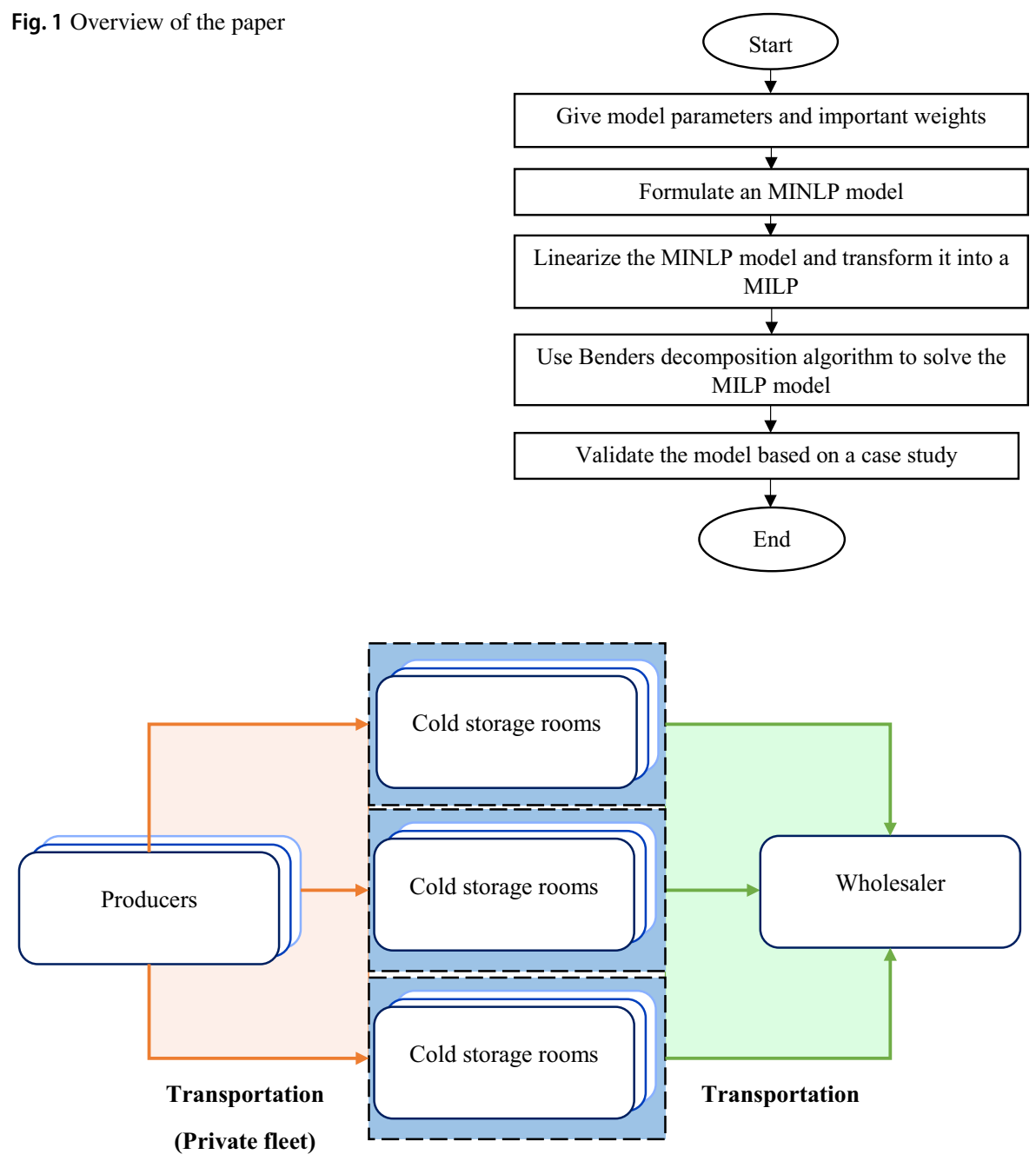

Warehouses

Fig. 2 Structure of the proposed agro-supply chain 
and busy harvest seasons require the producers to provide flexible operating by their private fleet. The processing operations should carry before the storage of products, by which products are sorted based on their varieties, sizes, etc. Then, each type of products regardless of their sizes, color, etc., are kept in a cold storage room, and the temperature conditions should be adjusted to optimize the shelf life of products. Afterward, the products are delivered to the wholesaler to satisfy customers' demands. Herein, transportation decisions are made by warehouses. In total, the proposed model aims to minimize the total cost of the agro-supply chain model while satisfying the constraints.

In order to formulate the mathematical model, the following assumptions are considered. In addition, indices, parameters, and decision variables are presented in Table 2.

\section{Assumptions}

- Shortages are not allowed.

- Three different quality grades are considered for products in orchards.

- Each of the quality grades for each variety of products imposes different amounts of losses.

- Each variety of products is available for purchase at a specific period. Therefore, they are purchased with sufficient quality to be stored in cold chambers in order to meet customers' demands for a while.

- Decisions on the type of temperature conditions should be made for each cold chamber in warehouses.

\subsection{Mixed-integer nonlinear model}

In this section, a MINLP model is presented as follows:

$$
\begin{aligned}
& \operatorname{MinZ}= \sum_{i \in I} \sum_{j \in J} \sum_{s \in S} \sum_{t \in T} a_{i t} C Q_{i j s} Q F_{i j s t} \\
&+\sum_{i \in I} \sum_{j \in J} \sum_{w \in W} \sum_{v \in V} \sum_{c \in C} \sum_{t \in T} C T_{j w} Q F W_{i j w v c t} \\
&+\sum_{i \in I} \sum_{w \in W} \sum_{v \in V} \sum_{c \in C} \sum_{k \in K} \sum_{t \in T} \sum_{g \in T E_{i w v c t}} P_{w} Q W_{i w v c k t g}+\sum_{w \in W} C W_{w} A W_{w} \\
&+\sum_{k \in K} \sum_{t \in T} C F T_{k} y_{k t}+\sum_{i \in I} \sum_{w \in W} \sum_{v \in V} \sum_{c \in C} \sum_{t \in T} \sum_{g \in T E_{i w v c t}} H W_{c} I_{i w v c t g} \\
& \sum_{j} \sum_{s} a_{i t} W E P_{i j s} Q F_{i j s t} \geq \alpha_{i} \sum_{j} \sum_{s} a_{i t} Q F_{i j s t} \forall i \in I ; t \in T
\end{aligned}
$$


Table 2 Indices, parameters, and decision variables

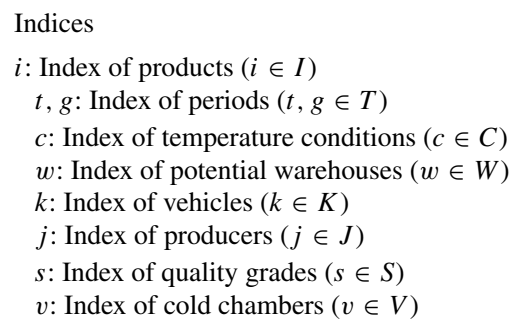

\section{Parameters}

$C T_{j w}$ : Operational costs to transport products from producer $j$ to warehouse $w$

$P_{w}$ : Operational costs in warehouse $w$

$H W_{c}$ : Holding cost of products under temperature condition $c$

$C F T_{k}$ : Fixed cost of transporting products by vehicle type $k$

$C W_{w}$ : Fixed cost of opening a warehouse at location $w$

$W_{w}$ : Available capacity of a cold chamber in warehouse $w$

$F W_{w}$ : Number of available cold chambers in a warehouse if it is opened at location w

$C V_{k}$ : Transportation capacity of vehicle type $k$

$C Q_{i j s}$ : Purchase cost of product $i$ with quality grade $s$ supplied by producer $j$

$T_{i c}$ : Shelf life of product $i$ under temperature condition $c$

$D_{i t}$ : Quantity of demand for product $i$ in period $t$

$L F_{i j s}$ : Loss factor of product $i$ with quality grade $s$ purchased from producer $j$

$C F_{i}$ : Supply capacity of product type $i$

$W E P_{i j s}$ : Quality score of product $i$ with quality grade $s$ supplied by producer $j$

$a_{i t}$ : Binary parameter; 1 if product $i$ is available in period $t, 0$ otherwise

$\alpha_{i}$ : Minimum acceptable quality score for product $i$

\section{Variables}

$Q F_{i j s t}$ : Quantity of product $i$ with quality grade $s$ purchased from producer $j$ in period $t$

$L O S S_{i j s}$ : Quantity of product loss $i$ with quality grade $s$ purchased from producer $j$

$Q F W_{i j w v c t}$ : Quantity of product $i$ purchased from producer $j$ by warehouse $w$ to be kept in cold chamber $v$ under temperature condition $\mathrm{c}$ in period $t$

$Q W_{i w v c k t g}$ : Quantity of stored product $i$ in warehouse $w$ in cold chamber $v$ under temperature condition $c$ in period $g$ to deliver to the wholesaler by vehicle type $k$ in period $t$

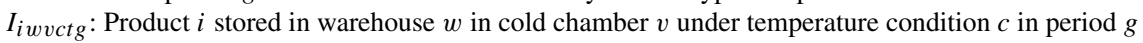
and remained until period $t$

$T E_{\text {iwvct }}$ : Storage time for product $i$ in warehouse $w$ in cold chamber $v$ under temperature condition $c$ in time period $t$

$y_{k t}$ : Number of vehicles $k$ for transporting products from warehouses to the wholesaler in period $t$

$A_{i j s t}$ : Binary variable, 1 if product $i$ with quality grade $s$ is supplied by producer $j$ in period $t, 0$ otherwise

$A C_{i w v c t}$ : Binary variable; 1 if cold chamber $v$ is turned on in warehouse $w$ under temperature condition $c$ in period $t, 0$ otherwise

$A W_{w}$ : Binary variable; 1 if a warehouse is opened at location $w, 0$ otherwise 


$$
\begin{aligned}
& \sum_{i} \sum_{c} A C_{i w v c t} \leq 1 \forall w \in W ; v \in V ; t \in T \\
& T E_{i w v c t} \leq T_{i c} A C_{i w v c t} \forall i \in I ; w \in W ; v \in V ; c \in C ; t \in T \text {; } \\
& \sum_{s} A_{i j s t} \leq 1 \forall i \in I ; j \in J ; t \in T \\
& Q F_{i j s t} \leq B I G A_{i j s t} \forall i \in I ; j \in J ; s \in S ; t \in T \\
& \sum_{w} \sum_{v} \sum_{c} Q F W_{i j w v c t} \leq \sum_{s} a_{i t}\left(Q F_{i j s t}-L O S S_{i j s t}\right) \forall i \in I ; j \in J ; t \in T \text {; } \\
& I_{i w v c t g}=I_{i w v c t-1 g}-\sum_{k} Q W_{i w v c k t g} \forall i \in I ; w \in W ; v \in V ; c \\
& \in C ; ; t \in T ; t-g \leq T E_{\text {icwvg }} \& g \in T E_{\text {icwg }} \& g<t \\
& I_{i w v c t g}=\sum_{j} Q F W_{i j w v c t}-\sum_{k} Q W_{i w v c k t g} \forall i \\
& \in I ; w \in W ; v \in V ; c \in C ; t \in T ; t=g \text {; } \\
& \sum_{j} Q F W_{i j w v c t}+\sum_{g(g<t)} I_{i w v c t-1 g} \leq W_{w} A C_{i w v c t} \forall w \in W ; v \in V ; c \in C ; i \in I ; t \in T ; \\
& \sum_{w} \sum_{v} \sum_{c} \sum_{k} \sum_{g} Q W_{i w v c k t g(g \leq t)} \geq D_{i t} \forall i \in I ; t \in T \\
& \sum_{i} \sum_{v} \sum_{c} A C_{i w v c t} \leq F W_{w} A W_{w} \forall w \in W ; t \in T ; \\
& \sum_{i} \sum_{w} \sum_{v} \sum_{c} \sum_{g} Q W_{i w v c k t g} \leq C V_{k} y_{k t} \forall k \in K ; t \in T \\
& \text { Loss }_{i j s t}=L F_{i j s} Q F_{i j s t} \forall i \in I ; j \in J ; s \in S ; t \in T \\
& \sum_{j} \sum_{s} a_{i t}\left(Q F_{i j s t}-L O S S_{i j s t}\right) \leq C F_{i} \forall i \in I ; t \in T \\
& Q F_{i j s t}, L O S S_{i j s t}, Q F W_{i j w v c t}, Q W_{i w v c k t g}, I_{i w v c t g} \\
& \geq 0 ; A W_{w}, A C_{i w v c t}, A_{i j s t} \in\{0,1\} ; y_{k t}, T E_{i w v c t} \in \text { Integer }^{+}
\end{aligned}
$$

The objective function (1) minimizes total cost consisting of six components, purchasing cost, operational costs including packing, laborers, and transportation costs (from producers to warehouses), operational costs in warehouses, fixed cost of opening warehouses, fixed cost of transportation (from warehouses to the wholesaler), 
inventory holding costs. Constraints (2) ensure that the purchased products are of satisfactory quality with reasonable prices. Constraints (3) ensure that only one type of product should be stored in each cold chamber under a specific temperature condition. Constraints (4) indicate the effects of temperature conditions on the shelf life of products. Constraints (5) ensure that products with the same quality grade are purchased from a producer. Constraints (6) show the relation between variables $A_{i j s t}$ and $Q F_{i j s t}$. Constraints (7) ensure that the amount of product in warehouses should not exceed the amount of purchased product. Equations (8) and (9) show the inventory level of products in warehouses where $g<t$ and $g=t$, respectively. Constraints (10) state the limited storage capacity of cold chambers in warehouses. Constraints (11) are the demand response. Constraints (12) represent the limitation in the number of cold chambers in a warehouse. Constraints (13) are capacity constraints of vehicles for transporting products from warehouses to the wholesaler. Equations (14) calculate the losses after purchasing products from producers. Constraints (15) indicate the available capacity of producers to supply product $i$. Constraints (16) define types of decision variables which are non-negative continuous, binary, or integer variables.

\subsection{Model linearization and reformulation}

In the proposed model, the upper limit of index $g$ is considered as a decision variable making the model nonlinear. In these cases, if-then decisions should be made to remove spoiled products from warehouses. Indeed, if the storage time exceeds the shelf life of products, the products should be removed from warehouses (If $t-g>$ $T E_{\text {iwvct }} \rightarrow I_{\text {iwvctg }}=0 ; Q W_{\text {iwvcktg }}=0$ ). Conversely, if the storage time is less than the shelf life of products, Eq. (8) are used to calculate the inventory level of products (If $t-g \leq T E_{\text {iwvct }} \rightarrow I_{\text {iwvctg }}=I_{\text {iwvct-1g }}-\sum_{k} Q W_{\text {iwvcktg }}$ ). In order to handle the nonlinear formulation and transform the MINLP model to MILP, a linearization technique is derived from Chen et al. (2010).

Consider the following nonlinear equivalents $\left(f(x) \leq b \rightarrow g_{j}(x) \leq b_{j}{ }^{\prime}\right)$ where $b$ and $b^{\prime}$ are the right-hand side of inequalities and $f(x)$ and $g_{j}(x)$ are the functions of variable $x$. In this case, if $f(x)$ is less than $b$, then $g_{j}(x) \leq b_{j}{ }^{\prime}$. The linearization of the if-then constraint is applied as follows:

$$
\begin{gathered}
-f(x)+b \leq B I G \cdot y \\
g_{j}(x)-b_{j}^{\prime} \leq B I G \cdot(1-y)
\end{gathered}
$$

where $B I G$ and $y$ are a positive big number and binary variable, respectively. Accordingly, the following equations are presented to linearize the if-then constraints in this paper.

$$
\begin{gathered}
t-g-T E_{i w v c g} \leq B I G \cdot Z_{i w v c t g} \\
I_{i w v c t g} \leq B I G \cdot\left(1-Z_{i w v c t g}\right)
\end{gathered}
$$




$$
\begin{gathered}
Q W_{i w v c k t g} \leq B I G \cdot\left(1-Z_{i w v c t g}\right) \\
I_{i w v c t g}-\left(I_{i w v c t-1 g}-\sum_{k} Q W_{i w v c k t g}\right) \leq B I G M \cdot\left(1-Z_{i w v c t g}\right)
\end{gathered}
$$

\section{Solution procedure}

The complexity of MILP problems rises by increasing the number of binary and integer variables, consequently, has a strong influence upon the computational time (Keshavarz-Ghorbani and Pasandideh 2021). Therefore, it is necessary to apply efficient solution procedures to overcome the computational burden. BD algorithm is known as one of the powerful algorithms to tackle the complexity of NP-hard problems. BD algorithm uses the concept of "divide and conquers" to reformulate the original problem into a master problem (MP) and dual subproblem (DSP) in a limited number of iterations. Nowadays, advantages of the BD algorithm, such as a limited number of iterations, less computational time, and guaranteed convergence for largescale problems, make it suitable for many optimization problems (e.g., Pishvaee et al. 2010; Shaw et al. 2016; Ghezavati et al. 2017; Kayvanfar et al. 2018; Babazadeh et al. 2019; Azadi et al. 2019; Mardan et al. 2019; Naderi et al. 2019; Alkaabneh et al. 2020; Gong and Zhang 2021).

The primary steps of the BD algorithm work iteratively by outer linearization and relaxation. In other words, the original problem is decomposed into an MP and subproblem (SP). The MP is a pure integer problem and includes integer and binary variables. The MP is solved and puts its solutions among the SP. In this step, the SP turns into DSP to make the feasible solution space independent from integer solutions. Then the DSP is solved, and its optimal bounded solutions/ unbounded solutions are added as feasibility/optimality cutting planes into the MP. This procedure reduces the computational time compared to the original problem. The convergence of BD comes from closing upper and lower bounds obtained from solving the DSP and MP, respectively. Figure 3 illustrates the steps of the iterative BD algorithm.

\subsection{Benders master problem}

To implement BD for the proposed model, the MP is called to minimize a part of the objective function associated with binary and integer variables with respect to constraints (3)-(5), and (12). That is a relaxation of the original problem to obtain lower bounds.

$$
\begin{aligned}
& \operatorname{Min} Z=\sum_{w \in W} C W_{w} A W_{w}+\sum_{k \in K} \sum_{t \in T} C F T_{k} y_{t k} \\
& \sum_{i} \sum_{c} A C_{i w v c t} \leq 1 \quad \forall w \in W ; v \in V ; t \in T
\end{aligned}
$$




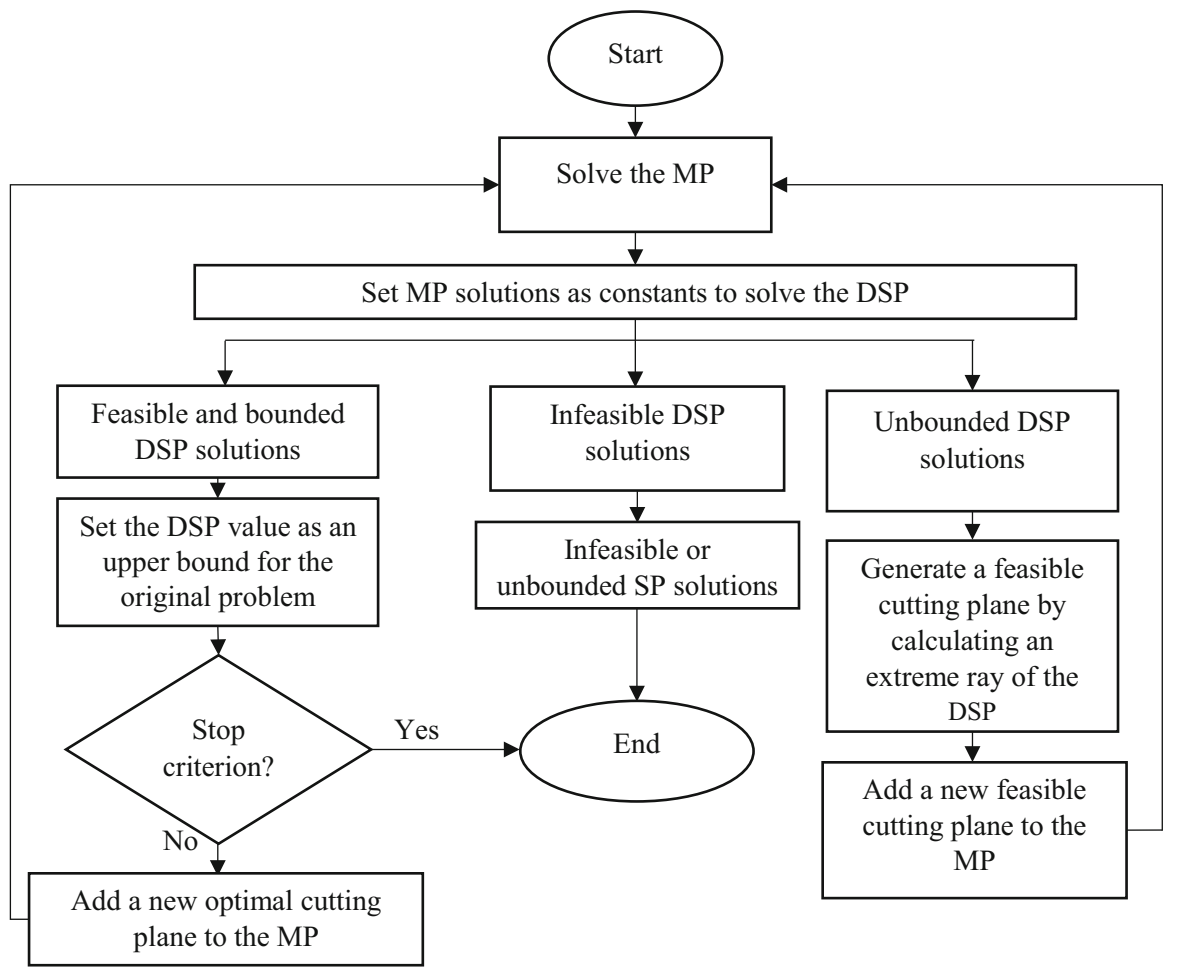

Fig. 3 The flowchart of BD

$$
\begin{aligned}
& T E_{i w v c t} \leq T_{i c} A C_{i w v c t} \quad \forall i \in I ; w \in W ; v \in V ; c \in C ; t \in T ; \\
& \sum_{s} A_{i j s t} \leq 1 \quad \forall i \in I ; j \in J ; t \in T ; \\
& \sum_{i} \sum_{v} \sum_{c} A C_{i w v c t} \leq F W_{w} A W_{w} \quad \forall w \in W ; t \in T \\
& A W_{w}, A C_{i w v c t}, A_{i j s t} \in\{0,1\} ; y_{k t}, T E_{i w v c t} \in \text { Integer }^{+}
\end{aligned}
$$

\subsection{Benders subproblem}

The SP includes a part of the objective function associated with continuous variables subject to constraints (2), (6)-(11), (13)-(15) that can be seen in Table 3. 
Table 3 Equations related to the SP and its corresponding dual variables

$\operatorname{MinZ}=\sum_{i \in I} \sum_{j \in J} \sum_{s \in S} \sum_{t \in T} a_{i t} C Q_{i j s} Q F_{i j s t}+$

$\sum_{i \in I} \sum_{w \in W} \sum_{v \in V} \sum_{c \in C} \sum_{k \in K} \sum_{t \in T} \sum_{g \in T E_{i c w t}} P_{w} Q W_{i w v c k t g}+$

$\sum_{i \in I} \sum_{j \in J} \sum_{w \in W} \sum_{v \in V} \sum_{c \in C} \sum_{t \in T} C T_{j w} Q F W_{i j w v c t}+$

$\sum_{i \in I} \sum_{w \in W} \sum_{v \in V} \sum_{c \in C} \sum_{t \in T} \sum_{g \in T E_{i c w t}} H W_{c} I_{i w v c t g}$

$Q F_{i j s t}<B I G A_{i j s t}$

$\beta 1_{i j s t}$

$\sum_{w} \sum_{v} \sum_{c} Q F W_{i j w v c t} \leq \sum_{s} a_{i t}\left(Q F_{i j s t}-L O S S_{i j s t}\right)$

$\beta 2_{i j t}$

$I_{i w v c t g}=I_{i w v c t-1 g}-\sum_{k} Q W_{\text {iwvcktg }}$

$\beta 3_{\text {iwvctg }}$

$I_{i w v c t g}=\sum_{j} Q F W_{i j w v c t}-\sum_{k} Q W_{i w v c k t g}$

$\beta 4_{i w v c t g}$

$\sum_{j} Q F W_{i j w v c t}+\sum_{g} I_{i w v c t-1 g} \leq W_{w} A C_{i w v c t}$

$\beta 5_{i w v c t}$

$\sum_{w} \sum_{v} \sum_{c} \sum_{k} \sum_{g} Q W_{\text {iwvcktg }} \geq D_{i t}$

$\beta 6_{i t}$

$\sum_{i} \sum_{w} \sum_{v} \sum_{c} \sum_{g} Q W_{\text {iwcktg }} \leq C V_{k} y_{k t}$

$\beta 7_{k t}$

$\sum_{j} \sum_{s} a_{i t}\left(Q F_{i j s t}-L O S S_{i j s t}\right) \leq C F_{i}$

$\beta 8_{i t}$

$\sum_{j} \sum_{s} a_{i t} W E P_{i j s} Q F_{i j s t} \geq \alpha_{i} \sum_{j} \sum_{s} a_{i t} Q F_{i j s t}$

$\beta 9_{i t}$

\subsection{Dual subproblem}

The DSP makes the feasible solution space independent from integer solutions and reaches the same objective function value as the SP. Therefore, the DSP is used instead of the SP to obtain upper bounds for the original problem. In this paper, the DSP is formulated as follows (Table 4):

The DSP and its corresponding variables are presented in Table 5.

After solving the DSP, an optimal or feasible cut is added to the MP according to Eq. (24) and an extreme ray of DSP. BD algorithm terminates whenever the stop criterion $\frac{U B_{\text {iter }}-L B_{\text {iter }}}{L B_{\text {iter }}} \leq \varepsilon$.

$$
Z_{\text {lower }} \geq \delta+\sum_{w \in W} C W_{w} A W_{w}+\sum_{k \in K} \sum_{t \in T} C F T_{k} y_{t k} \quad x \in S
$$

where $S$ is the feasible solution space of the MP, $\varepsilon$ is a small non-zero number. In addition, $U B_{i t e r}$ and $L B_{i t e r}$ are the upper and lower bounds obtained from solving DSP and MP, respectively. The pseudocode of $\mathrm{BD}$ is given as follows: 


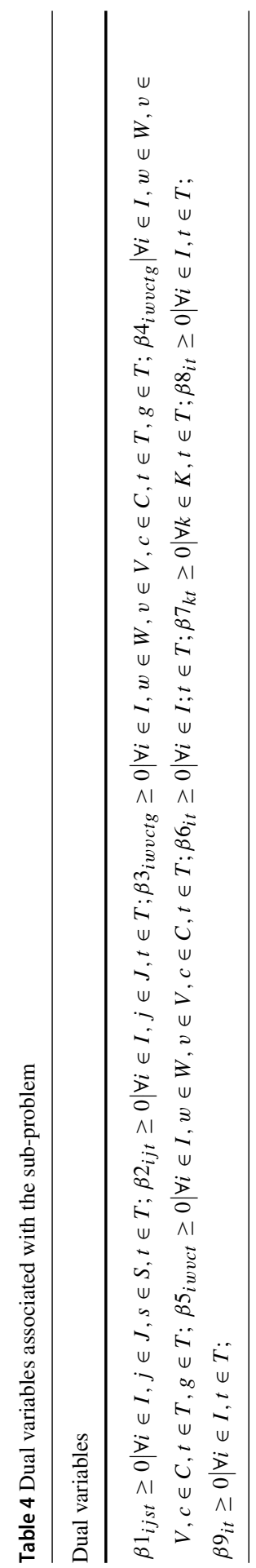


Table 5 The DSP and its corresponding variables

\begin{tabular}{ll}
\hline DSP & Variables \\
\hline$M a x \delta=\sum_{i} \sum_{j} \sum_{s} \sum_{t}\left(-B I G \bar{A}_{i j s t}\right) \beta 1_{i j s t}+$ & - \\
$\sum_{i} \sum_{w} \sum_{v} \sum_{c} \sum_{t}\left(-W_{w} \overline{A C}_{i w v c t}\right) \beta 5_{i w v c t}+\sum_{i} \sum_{t} D_{i t} \beta 6_{i t}+$ & \\
$\sum_{t} \sum_{k}-C V_{k} \bar{y}_{k t} \beta 8_{k t}+\sum_{i} \sum_{t}-C F_{i} \beta 9_{i t}$ & \\
$a_{i t}\left(-\beta 1_{i j s t}+\left(1-L F_{i j s}\right) \beta 2_{i j t}-\left(1-L F_{i j s}\right) \beta 8_{i t}+\left(W E P_{i j s}-\alpha_{i}\right) \beta 9_{i t}\right) \leq C Q_{i j s} s$ & $Q F_{i j s t}$ \\
$-\beta 2_{i j t}+\beta 4_{i w v c t g}-\beta 5_{i w v c t} \leq C T_{j w}$ & $Q F W_{i j w v c t}$ \\
$-\beta 3_{i w v c t g}+\beta 6_{i t}-\beta 7_{k t} \leq P_{w}$ & $Q W_{i w v c k t g} \mid t>g ;$ \\
& $t-g \leq \overline{T E}_{i w v c g}$ \\
$-\beta 4_{i w v c t g}+\beta 6_{i t}-\beta 7_{k t} \leq P_{w}$ & $Q W_{i w v c k t g} \mid t=g$ \\
$-\beta 3_{i w v c t g}+\beta 3_{i w v c t+1 g}-\beta 5_{i w v c t+1} \leq H W_{c}$ & $I_{i w v c t g} \mid \mathrm{t}>\mathrm{g} ;$ \\
$\beta 3_{i w v c t+1 g}+\beta 4_{i w v c t g}-\beta 5_{i w v c t+1} \leq H W_{c}$ & $t-g \leq \overline{T E}_{i w v c g}$ \\
\hline
\end{tabular}

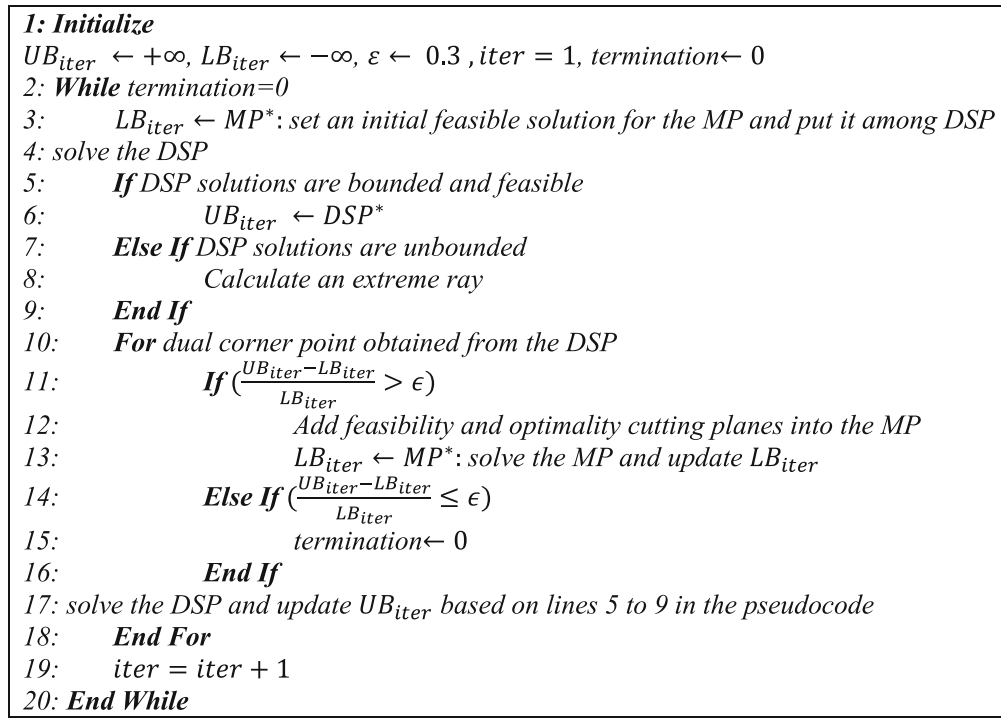

\section{Experimental results}

This section is presented in three parts. In the first part, numerical test problems, including small, medium, and large-sized instances, are executed for assessing the efficiency and applicability of the proposed model and solution approach. These test problems are randomly generated in Excel and presented in Tables 6 and 7. In the second part, the model is implemented in a case study in Iran to derive valuable insights. 
Table 6 Data generation functions

\begin{tabular}{ll}
\hline Parameters & Distribution functions \\
\hline$C T_{j w}$ & $\sim \operatorname{Uniform}(1,2)$ \\
$P_{w}$ & $\sim \operatorname{Uniform}(1,2)$ \\
$H W_{c}$ & $\sim \operatorname{Uniform}(1,2)$ \\
$C F T_{k}$ & $\sim \operatorname{Uniform}(1,2)$ \\
$F W_{w}$ & $\sim \operatorname{Uniform}(10,20)$ \\
$W_{w}$ & $\sim \operatorname{Uniform}(50,100)$ \\
$C W_{w}$ & $\sim \operatorname{Uniform}(1,2)$ \\
$C V_{k}$ & $\sim \operatorname{Uniform}(5,20)$ \\
$D_{i t}$ & $\sim \operatorname{Uniform}(10,100)$ \\
$C F_{i}$ & $\sim \operatorname{Uniform}(50,200)$ \\
$\alpha_{i}$ & $\sim \operatorname{Uniform}(0.6,0.8)$ \\
\hline
\end{tabular}

Table 7 Data generation functions for products with different quality grades

\begin{tabular}{llll}
\hline$s$ & $s 1$ & $s 2$ & $s 3$ \\
\hline$C Q_{i j s}$ & {$[900,1000)$} & {$[1000,1500)$} & {$[1500,2000]$} \\
$L F_{i j s}$ & {$[0.2,0.28]$} & {$[0.1,0.2)$} & {$[0.01,0.1)$} \\
\hline
\end{tabular}

Finally, a sensitivity analysis is carried out to help decision-makers determining key parameters which should be carefully tracked in the presented model.

\subsection{Validation of BD}

The presented MILP model and BD algorithm are coded in GAMS 27.2 optimization software and solved by a CPLEX solver. Both CPLEX solver and BD algorithm are executed on a computer with Intel ${ }^{@}$ Core $^{\mathrm{TM}}$ i7-CPU $2.20 \mathrm{GHz}$ and RAM 8.00 GB. Different sizes are presented in Table 8, and numerical results are presented in Table 9 , including optimal values of objective functions, the lower and upper bounds of BD, computational time, and gaps between upper and lower bounds of BD and CPLEX solver. BD algorithm continues once it reaches termination criteria which are as follows:

- The number of iterations reaches 200

- A relative gap between lower and upper bounds become less than $0.3 \%$

In addition, the CPLEX solver terminates once the absolute and relative gaps become less than 0.001 .

The results in Table 9 point out that the CPLEX solver reaches optimal solutions in less than one minute, whereas the computational time of $\mathrm{BD}$ is more than the CPLEX solver in several small sized-instances. As increasing the number of variables in medium sizes, the lower and upper bounds of BD converge to optimal solutions less than an hour, which is remarkably faster than the CPLEX solver. It should be noted, for 
Table 8 Generated small, medium, and large-sized instances

\begin{tabular}{llllllll}
\hline Instances & $I$ & $J$ & $W$ & $C$ & $S$ & $V$ & $K$ \\
\hline$P 1$ & 3 & 3 & 3 & 3 & 3 & 5 & 3 \\
$P 2$ & 4 & 3 & 3 & 3 & 3 & 5 & 3 \\
$P 3$ & 4 & 4 & 4 & 3 & 3 & 5 & 3 \\
$P 4$ & 3 & 8 & 5 & 3 & 3 & 5 & 3 \\
$P 5$ & 5 & 8 & 5 & 3 & 3 & 5 & 3 \\
$P 6$ & 8 & 8 & 5 & 3 & 3 & 5 & 3 \\
$P 7$ & 10 & 8 & 5 & 3 & 3 & 5 & 3 \\
$P 8$ & 10 & 10 & 8 & 3 & 3 & 5 & 3 \\
$P 9$ & 10 & 15 & 10 & 3 & 3 & 5 & 3 \\
$P 10$ & 12 & 15 & 12 & 3 & 3 & 5 & 3 \\
$P 11$ & 12 & 15 & 15 & 3 & 3 & 5 & 3 \\
$P 12$ & 15 & 15 & 20 & 3 & 3 & 10 & 3 \\
$P 13$ & 15 & 20 & 15 & 3 & 3 & 10 & 3 \\
$P 14$ & 20 & 20 & 10 & 3 & 3 & 10 & 3 \\
$P 15$ & 25 & 20 & 10 & 3 & 3 & 10 & 3 \\
$M i n$ & 3 & 3 & 3 & 3 & 3 & 5 & 3 \\
$M a x$ & 25 & 20 & 20 & 3 & 3 & 10 & 3 \\
\hline & & & & & & & \\
\hline
\end{tabular}

large sized-instances, the CPLEX solver cannot find optimal solutions and releases an "OUT OF MEMORY" error. In these cases, BD can be utilized to cope with complexity and reach optimal solutions in a reasonable time. Figure 4 demonstrates the changes of computational time for BD and CPLEX solver, in which BD outperforms CPLEX solver, especially in medium and large-sized instances. According to the results, the computational time of the CPLEX solver increases whatever the size of the problem increases. This affirms the efficiency of $\mathrm{BD}$ in solving the model in a reasonable time.

Besides, the average gap between the upper bounds of BD and the CPLEX solver is about 0.01684. It turns out BD converges to optimal solutions with a near-zero gap. In order to verify the efficiency of BD and its convergence, three instances in small, medium, and large sizes are derived from GAMS/CPLEX solver and presented as follows:

Figure 5 shows that BD provides optimality convergence in less than 17 iterations for a small-sized test (no. p5).

Figure 6 shows that BD provides optimality convergence in less than 12 iterations for a medium-sized test (no. p8). 


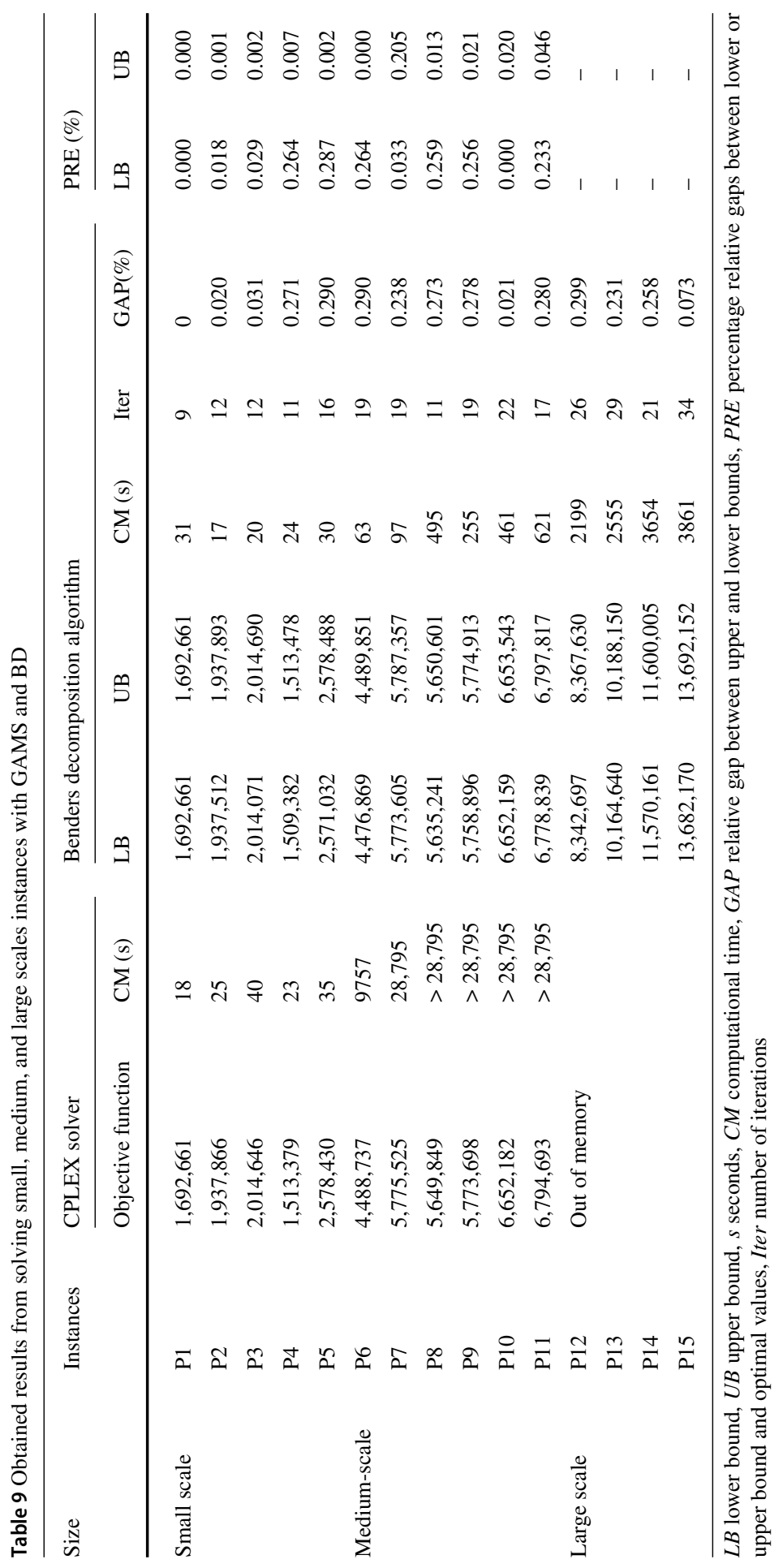




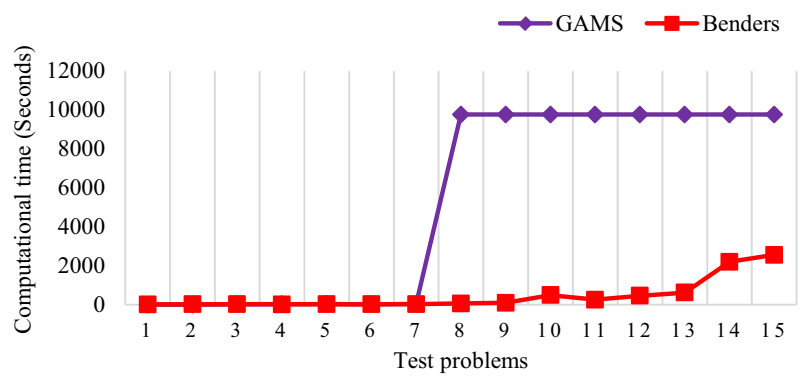

Fig. 4 Comparison of the computational time between GAMS and BD solvers

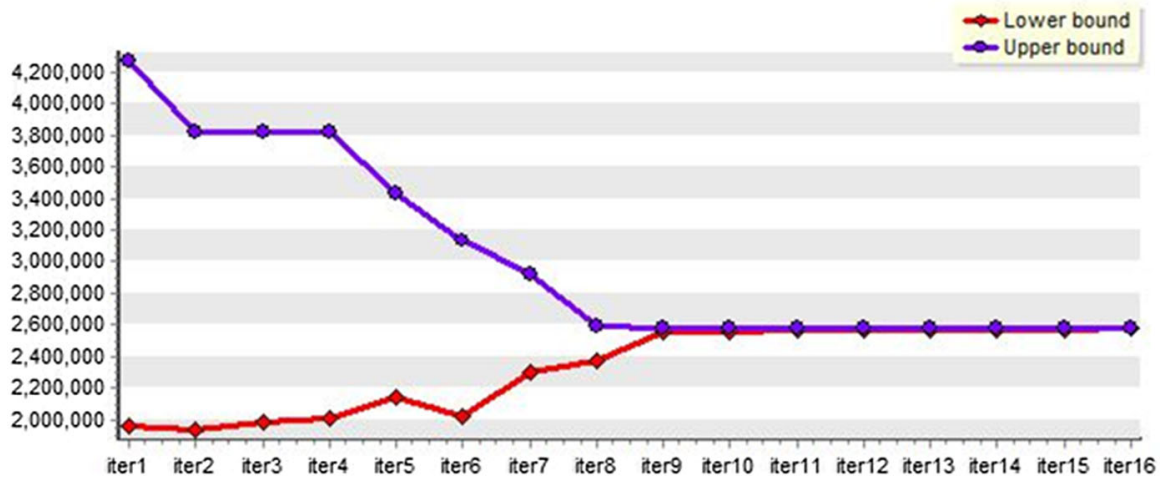

Fig. 5 The convergence of lower and upper bounds of BD for a small sized-instance (no. p5)

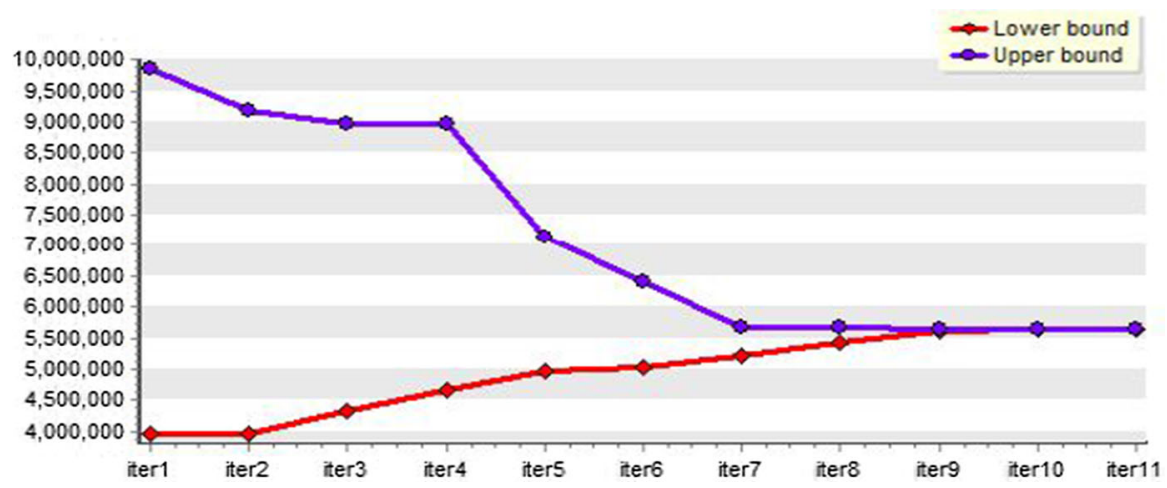

Fig. 6 The convergence of lower and upper bounds of BD for a medium sized-instance (no. p8) 


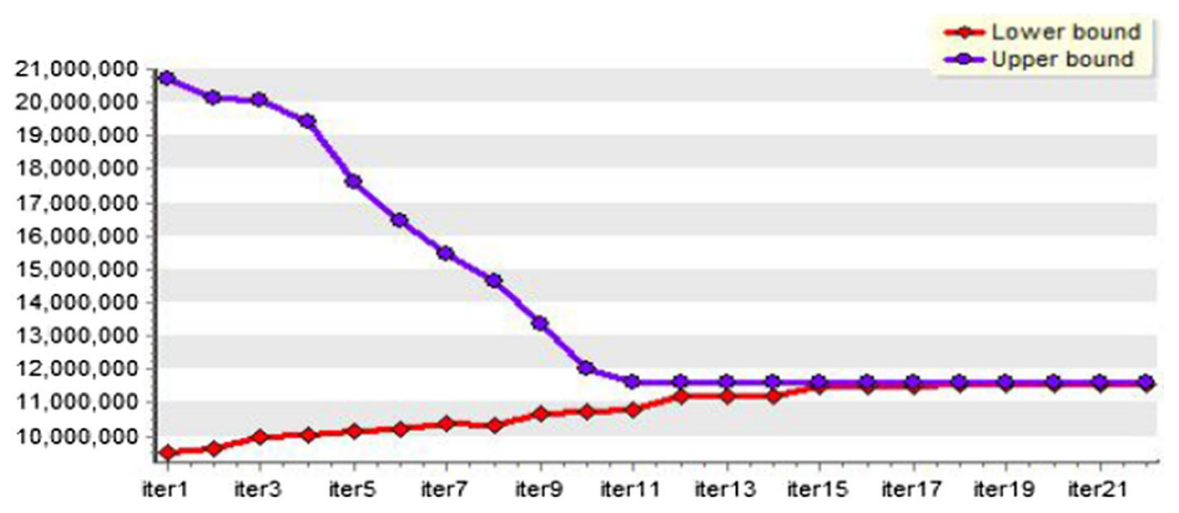

Fig. 7 The convergence of lower and upper bounds of BD for a large sized-instance (no. p14)

Figure 7 demonstrates that BD provides optimality convergence in less than 23 iterations for a large-sized test problem (no. p14).

\subsection{A case study}

This section devotes a discussion on an apple supply chain belonging to a domestic distributor in Iran to demonstrate the application of the proposed model. The company decides to establish warehouses at four potential locations, namely Tehran, Fars, Isfahan, and Razavi Khorasan, and activate cold chambers under proper temperature conditions. They often purchase different varieties of apples from producers who truck products by private vehicle fleet and located in Tehran, Alborz, Isfahan, West Azerbaijan, East Azerbaijan, and Semnan provinces. The company purchases good storage apples based on quality characters (i.e., texture, cosmetic appearance) to remain for a while in warehouses and satisfy the wholesaler's demands in different months of a year. Tables 10, 11, 12, 13, 14, 15 and 16 represent input data related to cold storage construction projects (www.damoon-co.com) and purchasing, transportation, and storage planning for eight months. The average demand is obtained based on historical data when the company faces shortages, and also demands should be met by any variety of apples. In addition, the purchasing cost of apples with different quality grades are available in fruit and vegetable fields organization in Iran, and information about temperature conditions in cold chambers are available in (https://pdfsara.ir/containerstorage-services-atmospheric-plan/).

The quality score of fruits supplied by different producers $\left(W E P_{i j s}\right)$ is calculated based on the minimum and maximum values of loss factor for a product with quality grade $s$ as follows:

$$
W E P_{i j s}=1-\frac{L F_{i j s}-M I L_{i s}}{M A L_{i s}-M I L_{i s}}
$$


Table 10 Input data related to the varieties of apples

\begin{tabular}{|c|c|c|c|c|c|c|c|c|c|c|c|c|}
\hline \multirow[t]{2}{*}{ Apples } & \multicolumn{3}{|c|}{$T_{i c}$} & \multirow[t]{2}{*}{$C F_{i} \times 10^{3}$ Ton } & \multicolumn{8}{|l|}{$a_{i t}$} \\
\hline & $c 1$ & $c 2$ & $c 3$ & & $t 1$ & $t 2$ & $t 3$ & $t 4$ & $t 5$ & $t 6$ & $t 7$ & $t 8$ \\
\hline Red delicious & 1 & 3 & 8 & 3.5 & - & - & - & - & 1 & 1 & - & - \\
\hline Golden delicious & 1 & 3 & 8 & 3.5 & - & - & - & - & 1 & 1 & - & - \\
\hline Golab & 1 & 0 & 0 & 4 & - & - & 1 & - & - & - & - & - \\
\hline Jonathan & 1 & 3 & 8 & 3 & - & - & - & - & 1 & 1 & - & - \\
\hline Granny Smith & 1 & 3 & 8 & 3 & - & - & - & - & 1 & 1 & - & - \\
\hline Fuji & 1 & 3 & 8 & 3 & - & - & - & - & 1 & 1 & - & - \\
\hline Qabaleh & 1 & 3 & 8 & 0.7 & - & - & - & - & 1 & 1 & - & - \\
\hline Baljei & 1 & 3 & 8 & 4 & 1 & - & - & - & - & - & - & - \\
\hline Soltani & 1 & 3 & 0 & 3 & - & 1 & - & - & - & - & - & - \\
\hline Mashhadi & 1 & 3 & 0 & 3.5 & - & 1 & 1 & - & - & - & - & - \\
\hline Gardeshiri & 1 & 0 & 0 & 3 & - & - & - & 1 & & - & - & - \\
\hline Asadkuhi & 1 & 3 & 0 & 3 & 1 & - & - & - & - & - & - & - \\
\hline
\end{tabular}

Table 11 Operational costs including transportation, packing, and sorting apples

\begin{tabular}{|c|c|c|c|c|}
\hline \multirow{2}{*}{$\begin{array}{l}C T_{j w}(\$) \\
\text { producers }\end{array}$} & \multicolumn{4}{|c|}{ Warehouses } \\
\hline & Tehran & Fars & Isfahan & Razavi Khorasar \\
\hline Tehran & 12.12 & 21.76 & 14.08 & 21.20 \\
\hline Alborz & 12.12 & 21.76 & 14.08 & 21.20 \\
\hline Isfahan & 14.36 & 14.34 & 12.10 & 26.01 \\
\hline West Azerbaijan & 19.34 & 29.26 & 29.18 & 16.06 \\
\hline East Azerbaijan & 6.51 & 2.48 & 19.31 & 26.20 \\
\hline Semnan & 21.2 & 27.14 & 26.01 & 5.04 \\
\hline
\end{tabular}

where $M I L_{i s}$ is the minimum loss factor for product $i$ with quality grade $s$ and $M A L_{i s}$ is the maximum loss factor for product $i$ with quality grade $s$. Equation (26) illustrates the quality score of producer $j 2$ supplying product $i 1$ with quality grade $s 3$, which equals 1 .

$$
W E P_{i j s}=1-\frac{0.06-0.06}{0.09-0.06}=1
$$


Table 12 Input data related to the vehicles

\begin{tabular}{lllll}
\hline & $k 1$ & $k 2$ & $k 3$ & $k 4$ \\
\hline$C V_{k}$ (Ton) & 5 & 10 & 15 & 25 \\
$C F T_{k}(\$)$ & 18.92 & 25.36 & 33.2 & 47.52 \\
\hline
\end{tabular}

Table 13 Input data related to warehouses

\begin{tabular}{lllll}
\hline & Tehran & Fars & Isfahan & Razavi Khorasan \\
\hline$C W_{w}\left(10^{4} \$\right)$ & 33.66 & 11.00 & 18.33 & 29.33 \\
$W_{w}($ Ton $)$ & 1000 & 500 & 500 & 1000 \\
$F W_{w}$ & 10 & 6 & 10 & 8 \\
$\left.P_{w}\left(10^{-4} \$\right)\right)$ & 3.31 & 0.99 & 1.66 & 2.64 \\
\hline
\end{tabular}

Table 14 Holding cost of apples under the different temperature conditions

\begin{tabular}{llll}
\hline & $c 1$ & $c 2$ & $c 3$ \\
\hline$H W_{c}(\$)$ & 29.4 & 50.4 & 159.92 \\
\hline
\end{tabular}

\subsection{Numerical results and discussion on a case study}

The model has 184,572 discrete variables, 330,049 continuous variables, and 642,748 equations for the case study and is implemented by both CPLEX solver and BD. Herein, the efficiency of the proposed model and related solutions have been discussed. The lower and upper bounds of BD become 25,289,297 and 25,289,302, respectively. In addition, the optimal solution is obtained by the CPLEX solver, which is $25,289,302$. The results indicate that $\mathrm{BD}$ converges to the optimal solution in a reasonable time. Figure 8 shows the convergence of $\mathrm{BD}$ for the case study.

First, producers who gain good scores are selected to supply products. The results show a $44 \%$ of apples are of first quality grade, $45 \%$ are of second grade, and $12 \%$ are of third quality grade. In this respect, the total cost of purchasing apples with different quality grades is about US\$996,000.

According to the results, it is beneficial to open warehouses in Tehran, Fars, and Isfahan provinces at a total cost of US\$ 6,600,000. In this case, the operational costs in warehouses become US\$ 147 . It is worth mentioning that the presented model is able to show the temperature condition in each cold chamber. Three temperature conditions are utilized for maintaining apples with different shelf life. Accordingly, $73.554 \%, 19.008 \%$, and $7.438 \%$ of products are kept under the temperature levels $c 1$, $c 2$, and $c 3$, respectively at a total cost of US\$17,520,000. In addition, operational costs, including laborer, packing, and transportation costs from producers to warehouses are about US $\$ 67,422$. Transportation cost between warehouses to the wholesaler is about 


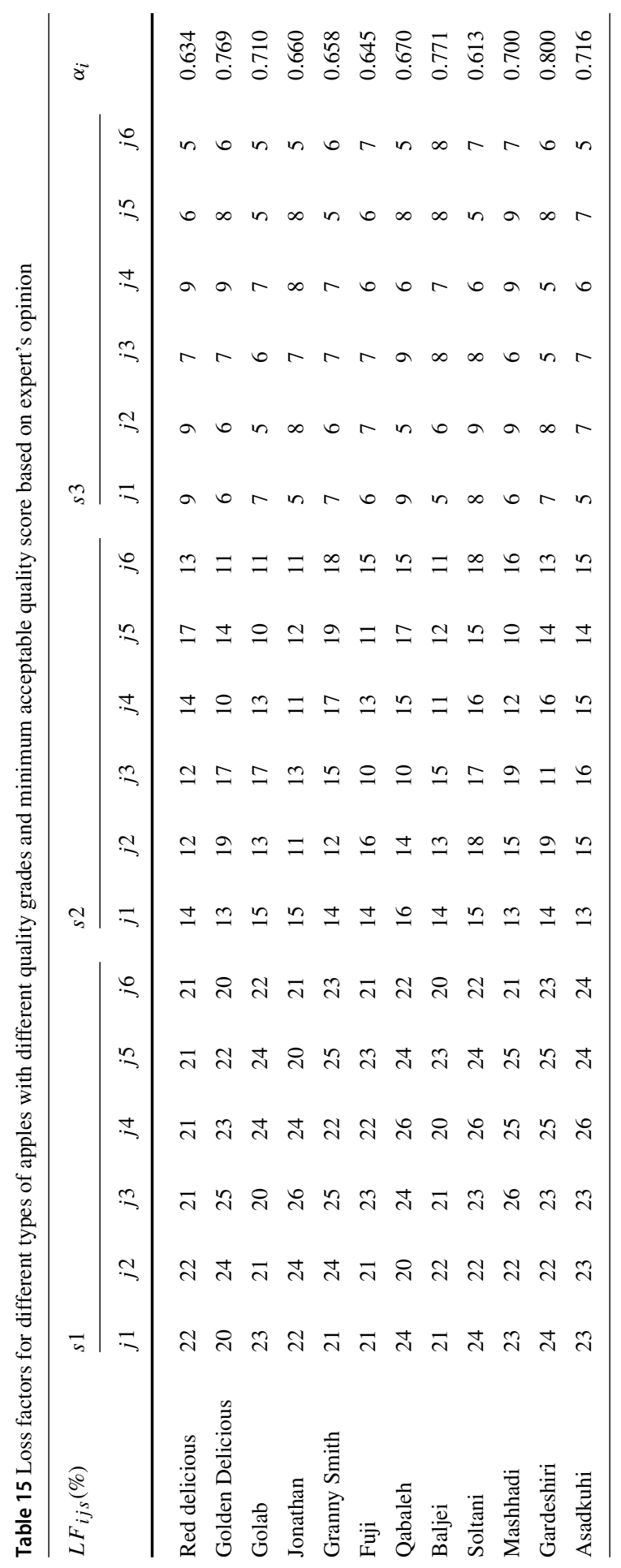


Table 16 Demand for different types of apples

\begin{tabular}{lllllllll}
\hline$D_{i t} \times 10^{3}$ (Ton) & $t 1$ & $t 2$ & $t 3$ & $t 4$ & $t 5$ & $t 6$ & $t 7$ & $t 8$ \\
\hline Red delicious & - & - & - & - & 1.000 & 0.900 & 1.560 & 1.400 \\
Golden Delicious & - & - & - & - & 0.650 & 0.780 & 1.000 & 1.080 \\
Golab & - & - & 2.530 & 1.438 & - & - & - & - \\
Jonathan & - & - & - & - & 1.350 & 1.246 & 1.321 & 1.350 \\
Granny Smith & - & - & - & - & 1.630 & 1.630 & 2.430 & 2.150 \\
Fuji & - & - & - & - & 1.450 & 1.525 & 2.350 & 2.096 \\
Qabaleh & - & - & - & - & 0.554 & 0.324 & 0.268 & - \\
Baljei & 1.500 & 1.000 & 1230 & 1.551 & 1.470 & 1.580 & - & - \\
Soltani & - & 1.450 & 1375 & - & - & - & - & - \\
Mashhadi & - & 1.250 & 1250 & 1.235 & - & - & - & - \\
Gardeshiri & - & - & - & 1.542 & 2.235 & - & - & - \\
Asadkuhi & 1.400 & 1.245 & 1.115 & - & - & - & - & - \\
$D_{t} \times 10^{3}$ (Ton) & 2.900 & 4.945 & 7.500 & 5.766 & 10.0339 & 7.985 & 8.929 & 8.076 \\
\hline
\end{tabular}

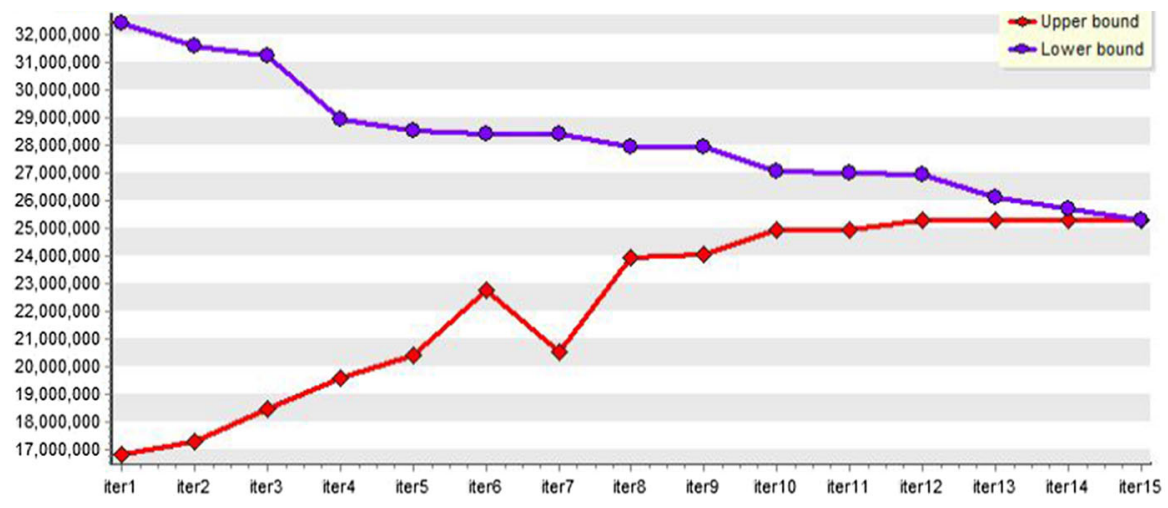

Fig. 8 The convergence of the lower and upper bounds of BD for the case study

US\$ 107,349 for 2262 trips, of which 1, 1, 3, and 2256 are performed by vehicles types $1,2,3$, and 4 , respectively.

\subsection{Sensitivity analysis}

Sensitivity analysis is performed on the case study to determine the behavior of the model by changing the main parameters. Clearly, there are many reasons affecting the selling price of products and may lead to fluctuation in demands. Changes in the selling price of products may be caused by specific economic conditions (e.g., economic impacts of COVID-19 outbreak), irreversible damage during production (e.g., frost, floods), etc. The customers' demand may be triggered by these factors or 
changes in taste and expectation, thereby becoming lesser or higher. From this point of view, it is vital to assess the model behavior during changes in parameters $D_{i t}$ and $C Q_{i j s}$. In addition, this paper gives four vehicle types for fruits transportation and two cold storage rooms for 500 or 1000 Tons of apples that may change. The containers of different capacities can easily fit onto different forms of trucks and provide other options for transportation planning. Besides, redesign of cold rooms of less capacity may affect the feasibility of the problem. Finally, two remaining parameters are inventory holding costs and operational costs, which may be changed by embracing new technologies, identifying inefficiencies and unused services, etc. Therefore, this section provides an overview of the changes and their impacts to revise the plan after the occurrence. The results are summarized in Table 17.

The results in Table 17 draw up the sensitivity of the model to parameters $D_{i t}$ and $H W_{c}$, where an increase of $25 \%$ in parameter $D_{i t}$ makes the model infeasible, and a decrease of $50 \%$ in parameter $D_{i t}$ reduce the total cost by $59 \%$. Indeed, it is

Table 17 The results of sensitivity analysis

\begin{tabular}{|c|c|c|c|}
\hline Parameters & Changes (\%) & Objective function values (\$) & Percentage changes (\%) \\
\hline \multirow[t]{4}{*}{$H W_{c}$} & 50 & $34,048,377$ & 34.63 \\
\hline & 25 & $29,668,828$ & 17.31 \\
\hline & -25 & $20,909,811$ & -17.31 \\
\hline & -50 & $16,530,329$ & -34.63 \\
\hline \multirow[t]{4}{*}{$W_{w}$} & 50 & $23,458,018$ & -7.24 \\
\hline & 25 & $24,281,249$ & -3.98 \\
\hline & -25 & $27,137,910$ & 7.31 \\
\hline & -50 & Infeasible & - \\
\hline \multirow[t]{4}{*}{$D_{i t}$} & 50 & Infeasible & - \\
\hline & 25 & Infeasible & - \\
\hline & -25 & $15,945,152$ & -36.94 \\
\hline & -50 & $10,358,746$ & -59.03 \\
\hline \multirow[t]{4}{*}{$C W_{w}$} & 50 & $28,589,282$ & 13.04 \\
\hline & 25 & $26,939,292$ & 6.52 \\
\hline & -25 & $23,639,312$ & -6.52 \\
\hline & -50 & $21,989,421$ & -13.04 \\
\hline \multirow[t]{4}{*}{$C Q_{i j s}$} & 50 & $25,785,923$ & 1.96 \\
\hline & 25 & $25,537,897$ & 0.98 \\
\hline & -25 & $25,040,161$ & -0.98 \\
\hline & -50 & $24,790,887$ & -1.97 \\
\hline \multirow[t]{4}{*}{$C V_{k}$} & 50 & $25,253,548$ & -0.14 \\
\hline & 25 & $25,267,852$ & -0.08 \\
\hline & -25 & $25,325,095$ & 0.14 \\
\hline & -50 & $25,396,595$ & 0.42 \\
\hline
\end{tabular}




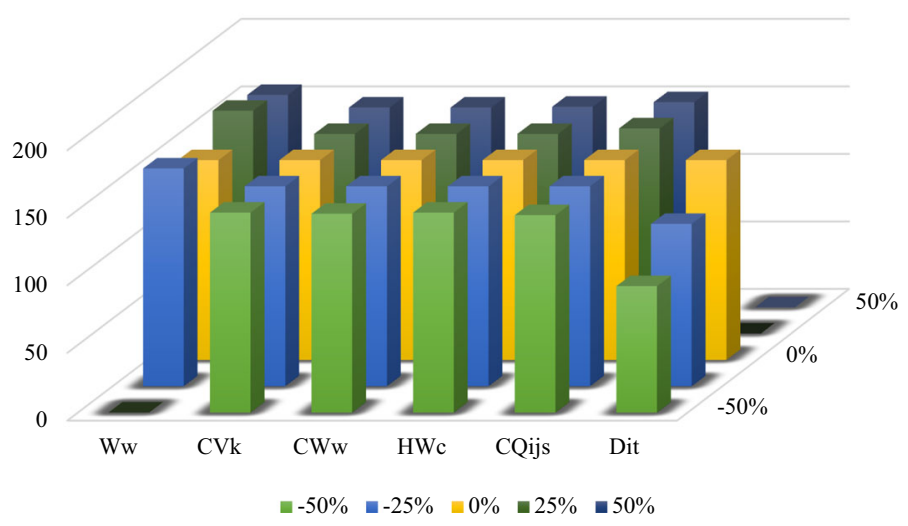

Fig. 9 Impacts of the changes on operational costs

necessary to adjust capacity to match it with the changes in demands. We suppose that the available capacity of warehouses or producers is not sufficient in response to increased demands, therefore, the model becomes infeasible. In this case, the capacity of either warehouses or producers may step up to meet the demand. In addition, it is expected to purchase fewer products and avoid overstocks when demand decreases. This may cause a decrease in purchasing costs, operational costs, and inventory holding costs. Besides, a 50\% increase in $H W_{c}$ leads to a $34.63 \%$ increase in the total cost. This indicates the importance of temperature condition adjustment and its impacts on the total cost.

For further investigation, the effects of the changes in parameters $H W c, C Q_{i j s}$, $D_{i t}$ on operational costs, inventory holding costs, and purchasing costs are presented as follows:

Figure 9 demonstrates the effects of the changes on operational costs.

- The changes in parameter $C Q_{i j s}$ have insignificant opposite impacts on the operational costs.

- The changes in parameter $D_{i t}$ have the greatest impacts on the operational costs, and a $25 \%$ decrease in demand leads to an $18 \%$ decrease in operational costs.

Figure 10 shows the impacts of the changes on inventory holding costs.

- A decrease in parameter $H W_{c}$ significantly reduces inventory holding costs. Indeed, a decrease of $25 \%$ in $H W_{c}$ leads to a $25 \%$ decrease in inventory holding costs.

- An increase in parameter $D_{i t}$ significantly affects the inventory holding costs, and a $25 \%$ decrease in $D_{i t}$ leads to a $36 \%$ decrease in inventory holding costs.

Figure 11 shows the impacts of the changes on purchase costs.

- Changes in parameter $D_{i t}$ have the greatest impacts on the total purchase cost. Indeed, a $25 \%$ decrease in $D_{i t}$ leads to a $27 \%$ decrease in total purchase cost.

- Total purchase cost increases by increasing the amount of parameter $C Q_{i j s}$. 


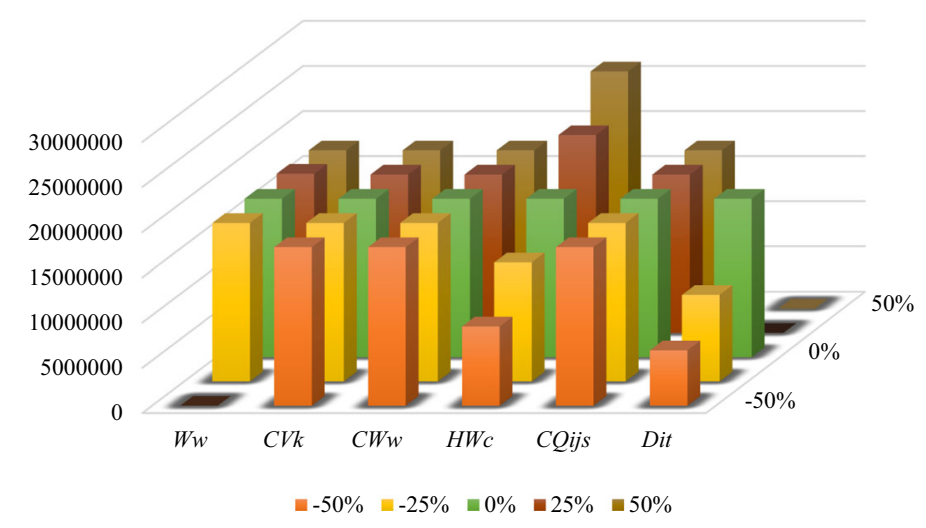

Fig. 10 Impacts of the changes on inventory holding costs

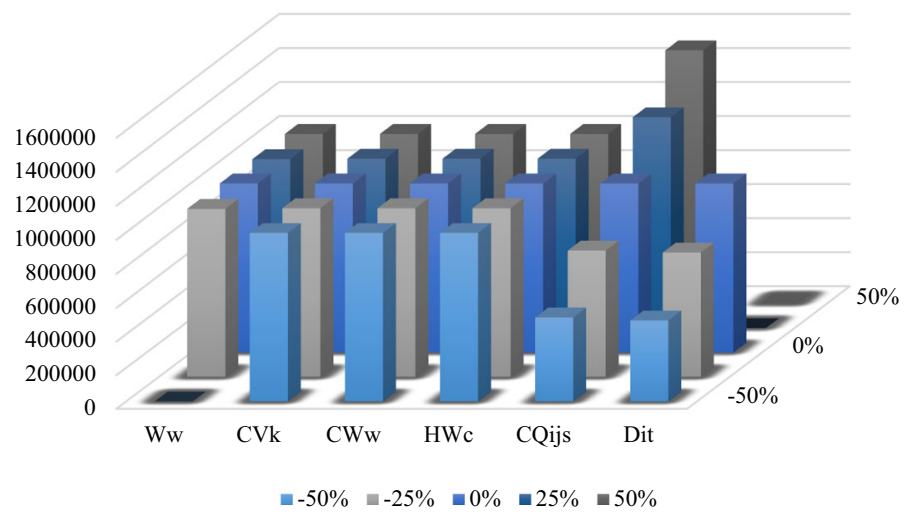

Fig. 11 Impacts of the changes on purchase costs

\section{Conclusion and future directions}

Nowadays, an appropriate configuration of agro-supply chains becomes all the more critical issues owing to specific characteristics of agro-products. Hence, in this paper, a multi-product multi-period MINLP model has been developed for an agro-supply chain contributing to purchase, facility location, and transportation decisions. Forasmuch as adjustment of temperature condition affects the quality of products and consequently their shelf lives, this study determines proper temperature conditions based on the varieties of products. This model aims to minimize the total cost of the supply chain considering several operational constraints.

The primary step to solve the proposed model is to linearize the MINLP model and reformulate it into a MILP problem. Then, three sets of numerical examples, small, medium, and large sizes, have been presented to evaluate the performance of the mathematical model. The problem was coded in GAMS software and executed 
for small and medium test problems. Whatever the size of the problem increases, the computational time increases and the problem becomes difficult to solve. Therefore, BD algorithm has been implemented to tackle the complexity of the problem. Then GAMS/CPLEX solver and BD were compared in terms of objective function values and computational time. The results depict the convergence of BD to optimal solutions in a reasonable time.

Afterward, a precise assessment of the model was carried out by implementing a real case study. The results turn out the optimal purchase decisions of required quality score to be kept for a long time and number of cold chambers under proper temperature conditions. In addition, a sensitivity analysis was performed on the main parameters to predict the effects of the variations.

For future research, first, the model can be revisited for conditions under uncertain demands, thereby proposing efficient scenarios to overcome their associated shortage. Another possible extension of the model is to analyze purchase decisions under discount. This paper assumes only the products of the required quality for long time storage should be purchased. However, a new framework is promising to accommodate the distribution of products with short and long shelf lives that need precise inventory management.

\section{Appendix}

\begin{tabular}{ll}
\hline Abbreviation & Description \\
\hline MINLP & Mixed-integer nonlinear programming \\
BD & Benders decomposition \\
MILP & Mixed-integer linear programming \\
MP & Master problem \\
SP & Subproblem \\
DSP & Dual sub-problem \\
SLD & Decision on shelf life \\
CFD & Decision on cold storage facilities \\
\hline
\end{tabular}

\section{References}

Ahumada O, Villalobos JR (2009) Application of planning models in the agri-food supply chain: a review. Eur J Oper Res 196(1):1-20. https://doi.org/10.1016/j.ejor.2008.02.014

Alkaabneh F, Diabat A, Gao HO (2020) Benders decomposition for the inventory vehicle routing problem with perishable products and environmental costs. Comput Oper Res 113:104751. https://doi. org/10.1016/j.cor.2019.07.009

Allaoui H, Guo Y, Choudhary A, Bloemhof J (2018) Sustainable agro-food supply chain design using two-stage hybrid multi-objective decision-making approach. Comput Oper Res 89:369-384. https:// doi.org/10.1016/j.cor.2016.10.012 
Aung MM, Chang YS (2014) Temperature management for the quality assurance of a perishable food supply chain. Food Control 40:198-207. https://doi.org/10.1016/j.foodcont.2013.11.016

Azadi Z, Eksioglu SD, Eksioglu B, Palak G (2019) Stochastic optimization models for joint pricing and inventory replenishment of perishable products. Comput Ind Eng 127:625-642. https://doi. org/10.1016/j.cie.2018.11.004

Babazadeh R, Ghaderi H, Pishvaee MS (2019) A benders-local branching algorithm for second-generation biodiesel supply chain network design under epistemic uncertainty. Comput Chem Eng 124:364-380. https://doi.org/10.1016/j.compchemeng.2019.01.013

Banasik A, Kanellopoulos A, Claassen GDH, Bloemhof-Ruwaard JM, van der Vorst JGAJ (2017) Closing loops in agricultural supply chains using multi-objective optimization: a case study of an industrial mushroom supply chain. Int J Prod Econ 183:409-420. https://doi.org/10.1016/j.ijpe.2016.08.012

Chen D, Batson R, Dang Y (2010) Applied integer programming: modeling and solution. Wiley, London

Coelho LC, Laporte G (2014) Optimal joint replenishment, delivery and inventory management policies for perishable products. Comput Oper Res 47:42-52. https://doi.org/10.1016/j.cor.2014.01.013

Ganesh Kumar C, Murugaiyan P, Madanmohan G (2017) Agri-food supply chain management: literature review. Intell Inf Manag 9:68-96. https://doi.org/10.4236/iim.2017.92004

Ghezavati V, Hooshyar S, Tavakkoli-Moghaddam R (2017) A Benders' decomposition algorithm for optimizing distribution of perishable products considering postharvest biological behavior in agri-food supply chain: a case study of tomato. CEJOR 25(1):29-54. https://doi.org/10.1007/s10100-015-0418-3

Gong H, Zhang Z-H (2021) Benders decomposition for the distributionally robust optimization of pricing and reverse logistics network design in remanufacturing systems. Eur J Oper Res. https://doi.org/10.1016/ j.ejor.2021.04.039

Jonkman J, Barbosa-Póvoa AP, Bloemhof JM (2019) Integrating harvesting decisions in the design of agro-food supply chains. Eur J Oper Res 276(1):247-258

Kayvanfar V, Husseini SM, Sajadieh MS, Karimi B (2018) A multi-echelon multi-product stochastic model to supply chain of small-and-medium enterprises in industrial clusters. Comput Ind Eng 115:69-79. https://doi.org/10.1016/j.cie.2017.11.003

Keshavarz-Ghorbani F, Pasandideh SHR (2021) A Lagrangian relaxation algorithm for optimizing a biobjective agro-supply chain model considering $\mathrm{CO}_{2}$ emissions. Ann Oper Res. https://doi.org/10.1007/ s10479-021-03936-1

Liu H, Zhang J, Zhou C, Ru Y (2018) Optimal purchase and inventory retrieval policies for perishable seasonal agricultural products. Omega 79:133-145. https://doi.org/10.1016/j.omega.2017.08.006

Liu L, Wang H, Xing S (2019) Optimization of distribution planning for agricultural products in logistics based on degree of maturity. Comput Electron Agric 160:1-7. https://doi.org/10.1016/j. compag.2019.02.030

Mardan E, Govindan K, Mina H, Gholami-Zanjani SM (2019) An accelerated benders decomposition algorithm for a bi-objective green closed loop supply chain network design problem. J Clean Prod 235:1499-1514. https://doi.org/10.1016/j.jclepro.2019.06.187

Morganti E, Gonzalez-Feliu J (2015) City logistics for perishable products. The case of the Parma's food hub. Case Stud Transp Policy 3(2):120-128

Morton LW, Bitto EA, Oakland MJ, Sand M (2008) Accessing food resources: rural and urban patterns of giving and getting food. Agric Hum Values 25(1):107-119. https://doi.org/10.1007/s10460-007-9095-8

Naderi B, Govindan K, Soleimani H (2019) A Benders decomposition approach for a real case supply chain network design with capacity acquisition and transporter planning: wheat distribution network. Ann Oper Res. https://doi.org/10.1007/s10479-019-03137-x

Onggo BS, Panadero J, Corlu CG, Juan AA (2019) Agri-food supply chains with stochastic demands: a multiperiod inventory routing problem with perishable products. Simul Model Pract Theory 97:101970. https://doi.org/10.1016/j.simpat.2019.101970

Orjuela-Castro JA, Sanabria-Coronado LA, Peralta-Lozano AM (2017) Coupling facility location models in the supply chain of perishable fruits. Res Transp Bus Manag 24:73-80. https://doi.org/10.1016/j. rtbm.2017.08.002

Otchia CS (2014) Agricultural modernization, structural change and pro-poor growth: policy options for the Democratic Republic of Congo. J Econ Struct 3(1):8

Paam P, Berretta R, Heydar M, Middleton RH, García-Flores R, Juliano P (2016) Planning models to optimize the agri-fresh food supply chain for loss minimization: a review. In: Reference module in food science, 1st edn. Elsevier. https://doi.org/10.1016/B978-0-08-100596-5.21069-X 
Paam P, Berretta R, Heydar M (2018) An integrated loss-based optimization model for apple supply chain. In: Operations research proceedings 2017. Springer, pp 663-669

Paam P, Berretta R, Heydar M, García-Flores R (2019) The impact of inventory management on economic and environmental sustainability in the apple industry. Comput Electron Agric 163:104848. https:// doi.org/10.1016/j.compag.2019.06.003

Pishvaee MS, Farahani RZ, Dullaert W (2010) A memetic algorithm for bi-objective integrated forward/reverse logistics network design. Comput Oper Res 37(6):1100-1112. https://doi.org/10.1016/ j.cor.2009.09.018

Shaw K, Irfan M, Shankar R, Yadav SS (2016) Low carbon chance constrained supply chain network design problem: a Benders decomposition based approach. Comput Ind Eng 98:483-497. https://doi. org/10.1016/j.cie.2016.06.011

Soto-Silva WE, González-Araya MC, Oliva-Fernández MA, Plà-Aragonés LM (2017) Optimizing fresh food logistics for processing: application for a large Chilean apple supply chain. Comput Electron Agric 136:42-57. https://doi.org/10.1016/j.compag.2017.02.020

Validi S, Bhattacharya A, Byrne P (2014) A case analysis of a sustainable food supply chain distribution system—a multi-objective approach. Int J Prod Econ 152:71-87. https://doi.org/10.1016/j. ijpe.2014.02.003

Widodo KH, Nagasawa H, Morizawa K, Ota M (2006) A periodical flowering-harvesting model for delivering agricultural fresh products. Eur J Oper Res 170(1):24-43. https://doi.org/10.1016/j. ejor.2004.05.024

Yu Y, Xiao T (2017) Pricing and cold-chain service level decisions in a fresh agri-products supply chain with logistics outsourcing. Comput Ind Eng 111:56-66. https://doi.org/10.1016/j.cie.2017.07.001

Yu Y, Xiao T, Feng Z (2019) Price and cold-chain service decisions versus integration in a fresh agri-product supply chain with competing retailers. Ann Oper Res 287(1):1-29

Publisher's Note Springer Nature remains neutral with regard to jurisdictional claims in published maps and institutional affiliations. 